\title{
ANNOTATIONS
}

\section{Blindness due to a rare form of Encephalitis}

Two articles in a recent number of Brain call attention to a very rare condition of the central nervous system in which occur symptoms of great interest to ophthalmic surgeons. The first article is by Dr. Bouman of Amsterdam, and the second by Drs. Collier and Greenfield, National Hospital, Queen Square. The disease with which they deal has evidently been known in a somewhat obscure way for some time, but was first defined and identified as a clinical entity by Schilder in the year 1912, who gave it the name of encephalitis periaxialis diffusa. It is allied, on the one hand, to disseminated sclerosis, and seemingly, on the other hand, to a diffuse gliomatosis. Unlike disseminated sclerosis, however, it seems to commence in one focus and not in a series of isolated foci. It spreads from its initial focus diffusely in the white matter of the centrum ovale. In the preponderance of cases, it seems to commence in the white matter of the occipital lobes, and spreads from there into the white matter of the temporal lobes, parietal lobes and frontal lobes, so that, ultimately, the whole white matter of the cerebral hemispheres may be affected. The myelin sheaths are the portions of the white matter primarily affected, and in this respect it resembles disseminated sclerosis.

The predominant feature of interest to ophthalmic surgeons is the early onset of blindness, evidently usually commencing as a hemianopia, sometimes quadrantic, sometimes altitudinal, but gradually or rapidly becoming complete, and without any ophthalmoscopic changes in the majority of cases. In only a few cases of rapid onset slight degrees of papilloedema have been observed. In one or two cases, slight ocular-motor palsies have been noted, but these have not been a common symptom. The most characteristic symptoms, apart from the blindness, are the fairly rapid development of spastic paralyses (diplegias and quadriplegias) and progressive amentia. The disease usually occurs in childhood or early adult life and is progressive to a fatal termination within two or three years, the final stages being marked by complete spasticity and amentia.

\section{Cause of Myopia}

The discussion on the causes of myopia still proceeds and seems likely to continue for long enough. In a letter to the editor of the Lancet of October 25, 1924, Edtidge-Green repeats the statements made in his Arris and Gale lecture of 1921 that myopia 